\title{
Robotic Arm for the Easy Mobility of Amputees using EMG Signals
}

\author{
VishnuvarthananGovindaraj, PallikondaRajasekaranMurugan, Sakthivel Sankaran, Madhan \\ Balaji, Sofia Fazila, Sheik Hussain Beevi, Marikaniand Ananda Lakshmi
}

\begin{abstract}
A robotic arm is a Programmable mechanical arm to replicate the functions of human arm. They are widely used in industries. Human robot-controlled interfaces mainly focus on providing rehabilitation to amputees in order to overcome their impairment or disability leading them to live a normal life. The major objective of this paper is to develop a movable robotic arm controlled by EMG signals from the muscles of the upper limb. In this system, we mainly focus on providing a low 2-dimensional input derived from electromyography to move the arm. This work involves creating a prosthesis system that allows signals recorded directly from the human body. The arm is mainly divided into 2 sections, control section and moving section. Movable part contains the servo motor which is connected to the Arduino Nano board, and it helps in developing a motion in accordance with the EMG signals acquired from the body. Control part is the part which is controlled by the operation in accordance with the movement of the amputee mainly the initiation of the movement with respect to the threshold fixed in the coding. The major theme of the project is to provide an affordable and easily operable device that helps even the poor sections of the amputated society to lead a happier and normal life by mimicking the functionaries of the human arm in terms of both the physical, structural as well as functional aspects.
\end{abstract}

Index Terms: Electromyography, Robotic Arm, Prosthesis, Control Section, Moving Section, Amputees.

\section{INTRODUCTION}

A robotic arm [11] may be defined as a programmable mechanical arm to replicate the human arm function. Its structure is similar to that of a complex robot with a manipulator and an end-effector.

Revised Manuscript Received on December 22, 2018.

VishnuvarthananGovindaraj, Department of Biomedical Engineering, Kalasalingam Academy of Research and Education, Virudhunagar,Tamilnadu, India. (email: gvvarthanan@gmail.com)

PallikondaRajasekaranMurugan, Department of Electronics and communication Engineering, Kalasalingam Academy of Research and Education, Virudhunagar,Tamilnadu, India. (email: mpraja80@gmail.com)

Sakthivel Sankaran, Department of Biomedical Engineering, Kalasalingam Academy of Research and Education, Virudhunagar,Tamilnadu, India. (email: sakthivelsankaran1992@gmail.com).

R.Madhan Balaji, Department of Biomedical Engineering, Agni College of Technology, Chennai,Tamilnadu, India. (email: madhansan 7@gmail.com)

M.Sofia Fazila, Department of Biomedical Engineering, Kalasalingam

Academy of Research and Education, Virudhunagar,Tamilnadu, India.

V.Sheik Hussain Beevi, Department of Biomedical Engineering, Kalasalingam Academy of Research and Education, Virudhunagar,Tamilnadu, India.

M.Marikani, Department of Biomedical Engineering, Kalasalingam Academy of Research and Education, Virudhunagar,Tamilnadu, India.

K.Ananda Lakshmi, Department of Biomedical Engineering, Kalasalingam Academy of Research and Education, Virudhunagar,Tamilnadu, India.
The human arm mainly consists of 2 types of movements which are rotational as well as translational. A bionic arm will be able to produce these movements with a greater efficiency. Here, in this case, the links of the manipulator are connected with joints of amputated patients and thus allowing the latter movements to be made possible.

Here, it is the end effector which is used to perform the functions of human arm. In case of mechanical robots, it may be used to perform functions such as gripping, holding, spinning, and several other motions, based on their applications. The robotic arm, commonly known as prosthetic arm may serve as an essential weapon for amputees to replace a missing body part due to injury, infection or congenital factors. The design of a prosthetic hand is basically dependent on the functional needs and appearance. It is created by the coordinated work of health care professionals and prosthetists. The design may be achieved manually or with the help of designing software. One can achieve a 3D model with the help of the input parameters that has been fed to the system. It is also possible for us to check the efficiency and performance, without the need for real time designing, which seems to be cumbersome in nature. One of the commonly used software interfaces is the CAD software, which stands for computer aided design. This software is mainly used by professionals to visualize a three-dimensional model of the prosthetic arm [6].

Upper extremity prosthesis [4] is classified into several types based on part of the arm that is to be replaced. It is classified as forequarter, shoulder disarticulation, trans-humeral prosthesis, elbow disarticulation, trans-radial prosthesis, wrist disarticulation, full hand, partial hand, finger and partial finger prostheses. Trans-radial prosthesis is the process of replacing limb that is missing below the elbow. This upper limb prosthesis can be classified into three categories, which includes Passive devices, Body Powered devices and Externally Powered (myoelectric) devices. The Passive devices are highly specific in terms of their function and are mainly for cosmetic applications. The mechanism of action involved in myoelectric arm is achieved by sensing EMG Signals, through electrodes placed over muscles [1]. An action potential is triggered in this way, causing an artificial hand to initiate the required motion or movement. A trans-radial prosthetic arm is also called as BE, which stands for below elbow prosthesis.

The project is principally based on the usage of the EMGsignals from the muscles of the forelimb. EMG stands for Electromyography, which is recorded by an Electromyogram for monitoring the electrical activity of various muscles of our body, especially the skeletal muscles.The muscle cells 
produce an action potential when they are triggered by either an electric stimulus or a neurological stimulus. Externally the muscle cells are triggered, with the help of electricity or voltage that is applied in few $\mathrm{mV}$ ranges.

The neurological stimulus is produced, as a result of reflex actions, evoked by the Central Nervous System that is either the brain or spinal cord in our body. [4] The Electromyography provides vital information about the functioning of the various muscles in our body. It helps in re-education of the muscles. It is also used to detect medical abnormalities activation. The biomechanics of human arm is mainly centered on the movement of human arm. One of the main advantages of EMG signals in the biomedical industries is its contribution to the prosthetic devices. EMG can be obtained with the help of electrode placement that may be done either invasively or non-invasively. For more accuracy and precision of the EMG signals obtained from the muscles, needle electrodes are preferred. But, one of its major disadvantages is the level of discomfort it causes to the patient.

For obtaining the EMG signal from a particular muscle, a needle electrode may be inserted, whereas, in the other cases of obtaining the EMG for a cluster of muscle in a specific region like the biceps brachia, surface EMG electrodes (sEMG) [7] may be placed intact with the body over the muscle surface. The number of electrodes to be placed depends on its application. It may have either unipolar configuration or bipolar configuration. In the case of unipolar configuration, one active and one reference electrode are used. The reference electrode is placed on the bone so that there is no interference with the muscle signals. In case of bipolar configuration, there are two active electrode and one reference electrode. The biomedical signal is a collection of various electrical signals in different ranges that are obtained from an organ representing a physical variable of interest. The signal is a function of time and can also be described by means of other factors such as amplitude frequency and phase shift. The EMG signals are mainly produced due to the neuromuscular activities that lead to contraction and relaxation of the muscles and thus flexion and extension movements are initiated in case of muscles of the forelimb. It is also dependent on various physiological and anatomical properties of the muscle.

The major interference or disturbance acquired in the obtained EMG signals of the muscle is due to the motion artefacts and noise that is caused due to the cross talk between neighboring muscles [10]. The motor unit action potentials are used for the design of prosthetic device [11]. Whenever an action potential is generated as a result of external stimulus in a neuron, it gets transferred to the adjacent neurons via dendrites and axon [8]. In this way a group of muscles get activated that result in an action potential that initiates the movement of the calcium ions in and out of a membrane of the muscle cells, thus resulting in either contraction or relaxation. This is the basis for the diagnosis of neurological disorders and it helps to create appropriate algorithms for the analysis of nature and characteristics of the EMG signal, and their possible use in operating prosthetic devices.

\section{RELATED WORKS}

The EMG controlled Robotic arm has become an interesting topic of discussion in modern times. A Multitasking EMG controlled Robotic arm [5] enabled the amputees to meet their requirements at their own will. The main components of the system are the microcontrollers and the accelerometers. The microcontroller [2] controls the robotic arm with the help of input EMG signals derived from the body. The accelerometer [9] determines the position, and the posture of the arm should be interpretedproperly so that the arm moves accordingly. The robotic arm proves to be helpful for the people who are disabled to overcome their difficulties. The robotic arm works based on an actuation system that functions in accordance with the EMG signal generated. This prosthetic device helps in satisfying the needs of the users, thereby allowing them to execute complex tasks by themselves. The performance of the prosthetic hand was quite remarkable as it had attractive features like dexterity, appearance, and excellent physical strength. The DC motors [15] are used to drive the arm. For conditioning of EMG signal, MATLAB software tools have been used. The MATLAB [14] provides an algorithm for controlling the arm movements due to the variations in amplitude values obtained from the EMG signals. The microcontrollers provide the control operation by introducing control schemes necessary for generating the arm movements with respect to input EMG signals acquired. The electrodes placed on the arm, senses the changes in amplitude values of the EMG signal of the muscles during contraction and relaxation phases. The output EMG signals obtained are fed to the microcontrollers, which help the prosthetic arm to deliver the desired action. The robotic arm which was designed using acrylic sheets [12] had reduced weights and minimal side effects.In another work, they have presented a robotic arm with 5 degrees of freedom (DOFs), which is controlled by EMG signals [13]. The main components involved in this process are EMG sensors, flex sensors, control unit, and signal processing unit and servo motors. The EMG sensors are meant for interpreting muscle movements in the human arm, while the flex sensors are used to determine angular displacement movements in joints and elbow regions of the human arm. Therefore, the study of myoelectric prosthesis has made Human to Robotic Interactions much easier and simple.

\section{PROBLEM STATEMENT}

Amputation causes permanent disability for the affected individual that leads to a drastic change in functioning of their life and their appearance as well. This brings a great change in their aesthetic appearance making them feel low and suppressed in front of others. The situation change seems to be worsening in the case of lower limb amputees than the upper limb amputees, due to their inability to walk affecting their locomotion. The major causes of this amputation are due to vascular complications as seen in case of Diabetes or due to traumatic incidents. Amputation challenges the individuals to make an active participation in the society and also affects their functioning as an independent individual.

Due to these factors, they reach a depressed state. They also keep on longing for a better psychosocial support. The major focus of these

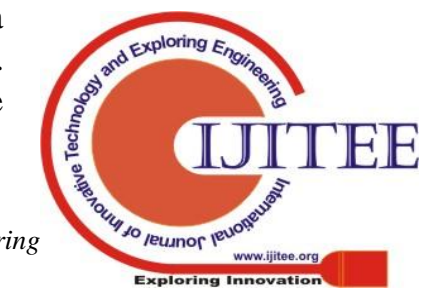


prosthetic devices is to provide rehabilitation goal in supporting the mobility of the amputees and thus helping in improving their quality of life. This will help them to regain back their confidence and thus lead a better life by taking active participation in the society.

\section{MOTIVATION}

We have been blessed with good health for leading a healthy life. Missing a body or losing it because of any trauma infection or accident and inability to regain it back is an indigestible situation, which is faced by a significant part of the population nowadays. The worst cases are those people who had lost their arm due to physical conditions such as accidents, as leading the rest of the life without a body part, once they had earlier, seems to be more troublesome, compared to those, who never had an idea of its functioning and importance since from their birth, which is seen in the case of congenital amputees. Keeping all these problems faced by them in our mind, we brought up the idea of designing a robotic arm that will help them overcome such disabilities and thus, making them to lead a better livelihood. Our goal is mainly to design a dexterous arm that will replicate the structural and functional aspects of the human arm, thereby giving the amputees a better look with no adjustments to their functioning when compared to the normal person.

\section{NEED ANALYSIS}

Amputated condition bought up great hurdles in the life of disabled individuals. The sour truth is that their inability to perform their daily routine tasks as well as social activities, in such a way as done before. When it comes to contribution given by the current prosthetic devices, they are not up to the expectation level of the disabled person. It does not help them completely to overcome their problems. They lack interfacing the prosthesis with human bio-signals. Lack of tactile feedback is also a major problem contributing to the poor working of these prosthetic devices.

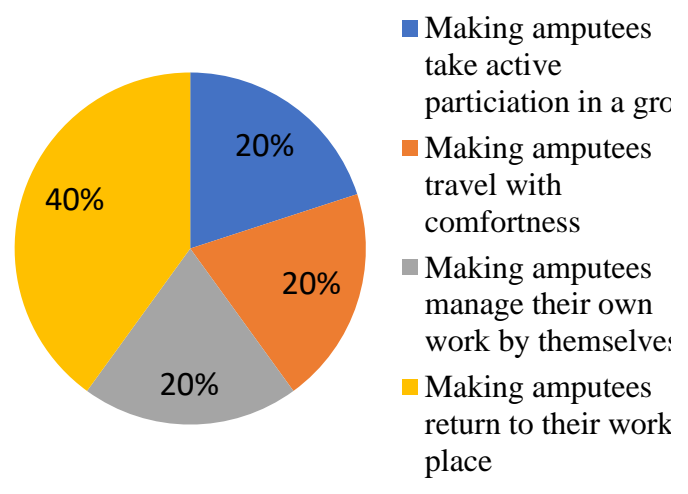

Fig. 1. Need Analysis

But, when it comes to device designed by us, it proves to be both cost effective as well as more effective in interfacing the software with hardware components thus initiating better movement in terms of both flexion and extension in the upper limb. The pie-chart shown below depicts the importance of this project to the amputated community based on the survey conducted from both amputated patients as well as orthopedic physicians.

\section{METHODOLOGY}

The project deals with introduction of the EMG signal from the body and that signal is responsible for the production of movement in the robotic arm, attached with servo motor and Arduino Nano. Both the hardware and software tools communicate with one another, in order to make the correct decision whether to move the arm or not, based upon the intention of the subject involved in the process. The project works with the help of an Arduino code that functions whenever an input EMG signal arrives. The code has threshold value that distinguishes between the different EMG values obtained with which the arm is able to produce flexion and extension movements accordingly. Initially, the EMG signal has to be acquired. For this purpose, we have to make use of surface EMG electrodes. Three electrodes are involved in this process. The active electrodes are placed on the mid muscle and end muscle regions of the biceps muscles respectively. The reference electrode is placed near to the bony surface area of the elbow.

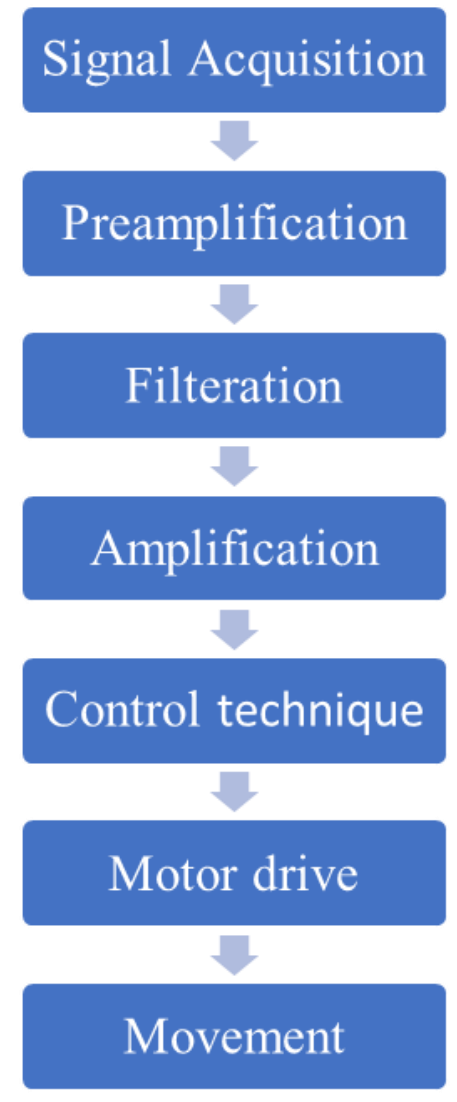

Fig. 2. Flow Diagram

Proper positioning of electrodes is necessary for the operation. At the same time, the electrodes that we have chosen should be capable of picking up the desired EMG signals more effectively.

Once we have done with this signal acquisition part, the next step is to process the raw EMG signal to get clear results at the end. The stages of EMG signal processing involve: pre-amplification, rectification, smoothening and post-amplification. By this way, the EMG signal conditioning process is carried out using suitable circuitry constructed on a 
breadboard. After this phase, our next work is to make the resultant EMG signal available for the Arduino Nano. The Arduino Nano works as a brain of the system that commands the robotic arm attached with it, to perform the desired actions.

An embedded C code program is written for controlling the operation of the arm movements. The code is then uploaded on the Arduino Nano, which is connected to a monitor. The Arduino Nano is connected with the external robotic arm through the servo motor. As the code runs, the servo drives the robotic arm to produce the desired movements, with the help of EMG signal values acquired from the body, during flexion and extension processes. Thus, by implementing the project, we can help the amputees to bring back their lost functionaries of their upper extremities, allowing them to do their day-to-day activities, with the help of robotic arm attached to their body part.

\section{CIRCUIT DIAGRAM}

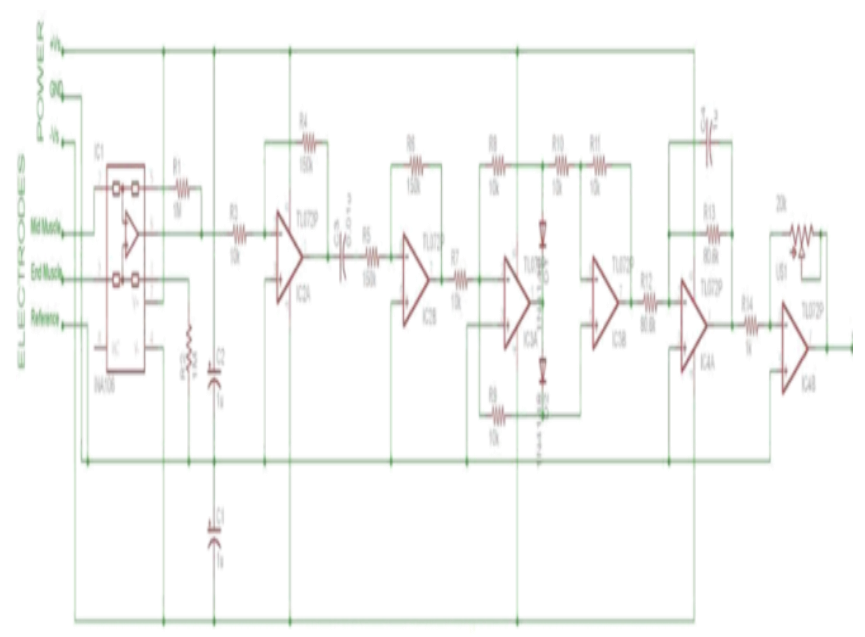

Fig. 3. EMG sensor circuit

\section{HARDWARE CIRCUIT EXPLANATION}

\section{Power Supply}

First of all, we need to connect two 9V batteries in series to produce $18 \mathrm{~V}$ of power supply to turn the circuit on.

\section{EMG Acquisition}

EMG signal is the measure of electrical activities of the muscles, caused due to electrical impulses generated by the nervous system of the body. The EMG signal is acquired, by using EMG electrodes placed over the biceps muscles. The reference electrode is placed on the elbow region.

\section{EMG Signal Processing}

i. Pre-amplification

This is done with the help of the INA106. INA106 is an instrumentation amplifier that is capable of amplifying (with an amplifier gain value of 110) the very small voltage difference between the two electrodes placed on the biceps muscles, by which the desired EMG signals are acquired.

\section{ii. Amplification}

Here, we use an inverting amplifier, TL072 that amplifies the EMG signal in an inverting manner with a gain of -15 .

$$
\text { iii. High pass filtering }
$$

An active high pass filter is built using two $150 \mathrm{k}$ ohm resistors and a 0.01 microfarad capacitor. Thishelps in removal of DC offset error and low frequency noise.

iv. Rectification

This process is made available by construction of a full wave rectifier using five $10 \mathrm{k}$ ohm resistors, two IN4148 diodes and a TL072 inverting amplifier IC.

v. Low pass filtering

An active low pass filter is designed by making use of a TL072 IC, two $82 \mathrm{k}$ ohm resistors, a $100 \mathrm{k} \Omega$ POT and a 1.0 microfarad capacitor. Low pass filtering helps in attenuating high frequency signals and it allows only low frequency signals to pass through the filter.

\section{vi. Post-amplification}

Since TL072 IC is an inverting amplifier, there is a chance for getting the output of low pass filtered signal in an inverting fashion. So, to avoid this, we need to use a $100 \mathrm{k} \Omega$ POT which helps in inverting the signal one more time. Generally, POT, also called as potentiometers, isa variable resistor that can be configured to a particular resistance value as per the user's needs. Initially, we set the POT value to a resistance value of $20 \mathrm{k} \Omega$.

\section{Circuit Protection}

For protecting the circuit from short circuit problems, we choose two 1.0 microfarad capacitors. This is achieved by connecting one of the 1.0 microfarad capacitor between the positive terminal of the battery and the GND, while the other 1.0 microfarad capacitor between the negative terminal of the battery and the GND.

\section{Electrode Cable Connection}

The electrode cables are connected in such a way that the mid muscle electrodes are connected to the pin 2 of INA106 IC, the end muscle electrodes are connected to pin 3 of INA106 IC and the reference electrode is connected to the GND connection, respectively.

\section{Establishing Connection with Arduino Nano}

By connecting the pin 1 of the last TL072 IC to the Analog pin 0 of the Arduino Nano, we can be able to establish a connection between Arduino Nano and the breadboard circuitry.

\section{Precautions to be followed}

Before giving the circuit connection, disconnect the batteries supply connection. Once the circuit has been built, make sure that the output voltage from the circuit is tolerable by the Analog pin of the Arduino Nano microcontrollers. Thus, one should be very careful while giving the circuit connection because even a small mistake could ruin the circuit.

\section{Servo Connection with Arduino}

The servo motor is connected to the Digital pin 4 of the Arduino Nano. The servo drives a mechanical robotic arm as per the desired EMG signal values obtained.

\section{Arduino Code}

The Arduino code is written on an embedded C platform. This code makes it possible to establish a connection between a natural arm and a robotic arm. We have used Arduino software, which is running on a Arduino Nano - Atmega328 board.

10. Pseudocode Include the servo library 
Initialize THRESHOLD for the EMG Signal acquired

Set the EMG_PIN to Analog pin 0

Set the SERVO_PIN to Digital pin 4

Create a servo variable SERVO_1

Set the BAUD RATE for serial data transmission

Attach the servo variable to the Digital pin 4

Assign a VALUE variable to read the Analog values of the EMG_PIN

IF the VALUE is greater than the THRESHOLD

THEN

The servo motor will turn to 170 degrees in clockwise direction, showing extension

ELSE

The servo motor will turn to 30 degrees in anticlockwise direction, showing flexion

Produce the resultant EMG values from the VALUE variable in serial monitor

\section{EXPERIMENTAL OUTPUT}

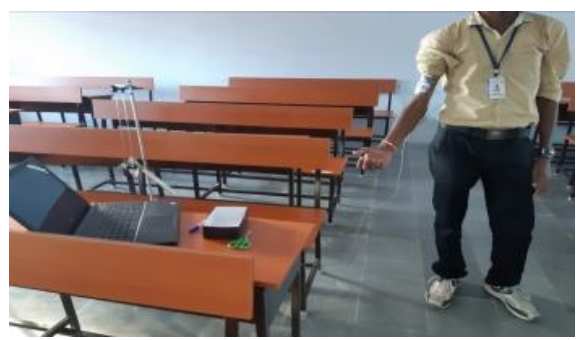

Fig. 4. Setup

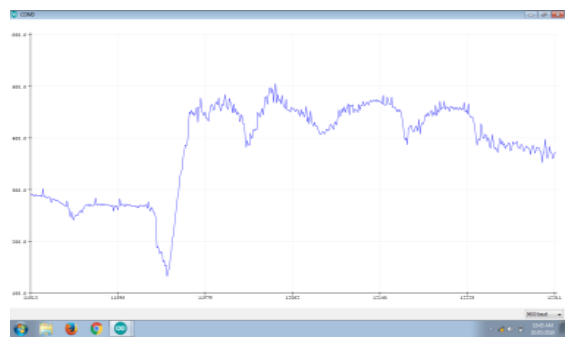

Fig. 5. EMG Graph

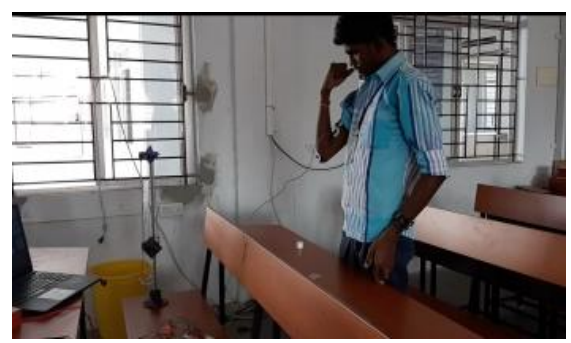

Fig. 6. Flexion

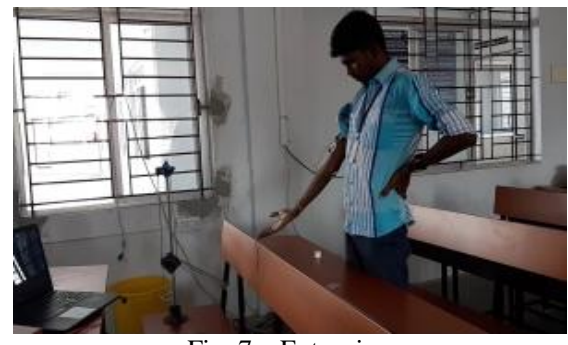

Fig. 7. Extension

\section{PERFORMANCE ANALYSIS}

\section{ACCURACY}

normal state

Muscle Fatigue

Time Period

Fig. 8. Accuracy

\section{SENSITIVITY}

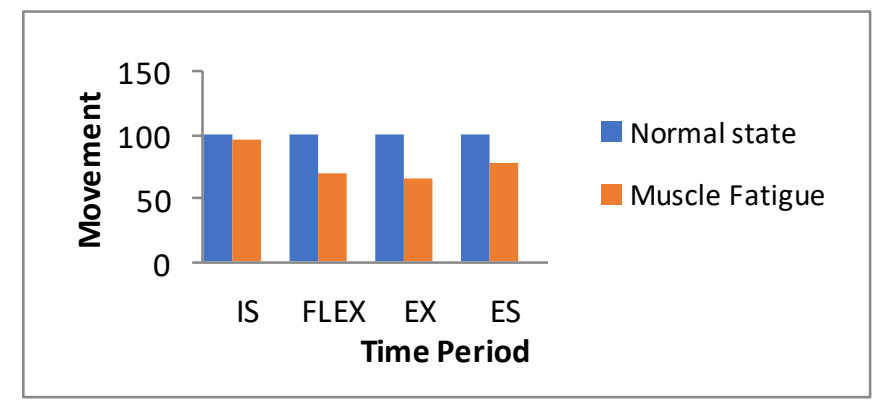

Fig. 9. Senstivity

Here, in this case, graph has been plotted for the initial state, Flexion, Extension and Equilibrium state, against time period, to indicate the performance of EMG signals measured from an individual,at normal and muscle fatigue conditions respectively. By this chart, we conclude that the hand movements fetched out of EMG signals is efficient at normal condition, but, in case of Muscle Fatigue condition, we could not able to get proper results due to stress applied over a long period of time in the muscle.

The developed system has significantly contributed near to $100 \%$ in terms of Accuracy and Sensitivity, when there is no loss of muscle strength,and this tends to ease the predicaments faced by upper-limb amputees.

\section{RESULTS}

A prototype mimicking the functions of the human arm were successfully designed, bringing out various gestures and motions for the betterment of the amputees, in order to accomplish the basic motions of the body, such as flexion and extension. This project works for improvement in quality of life of the amputees. We were able to accomplish 2 motions in the upper extremities. Flexion was obtained by anticlockwise rotation of the servomotor, and extension by clockwise rotation of the servomotor, which is attached to Arduino board. Moreover, we were able to design a circuit on a breadboard, which was looking so effective, compact and portable without many complications involved in its working process. The threshold could be fixed, and modified anytime in the Arduino program coding, based on user's requirement. Thus, we were able to design an easily accessible robotic arm for amputees, which would be affordable, even to the lower sections of the society.

\section{CONCLUSION}

In this Research paper, the input EMG Signals were taken from a healthy subject.The acquired EMG signals are then fed to the preamplifier for the amplification process. Next these signals are filtered in order to remove the artifacts and unwanted interferences. Then these are amplified and

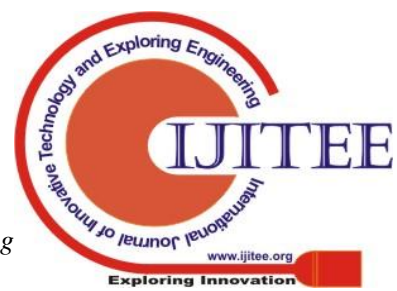


fed to the Arduino Nano, in order to activate the servo motor, to drive the motor either clockwise or anticlockwise, based on the strength of the received signal after the further signal processing. In our case, we have fixed the threshold as 400 . The threshold was chosen, based on the reading acquired from the serial monitor displayed during the Arduino coding execution.

The concept that we have presented in this paper is just the beginning for bringing out a final product, with high performance rate at minimal cost afforded for buying it.We got the inspiration of carrying out this project from a work, which was done based on developing of EMG controlled prosthetic arm, by students of Toc $\mathrm{H}$ Institute of Science and Technology, Kerala, which was priced at 2 lakhs INR. With the help of advanced technologies, like Artificial Intelligence, the efficiency of the arm motions could be improved further.

\section{FUTURE WORKS}

In future we have planned to develop our project in real time basis. We focus in bringing more than 2 motions that is abduction, adduction, and external rotation. We have also planned to develop the arm by a biocompatible material with grasping capabilities to bring out finger movements thus improving the efficiency of the arm. We also aim to develop more innovation techniques to design the arm in such a way that they mimic and exactly replicate the functions of human arm.

\section{REFERENCES}

1. [1]E. Cavallaro, et al. Based Model as a Myoprocessor for a Neural IEEE Int. Conf. Robot Automation 2005. pp. 4514-19.

2. [2] O. Fukuda, et al. A human-assisting manipulator teleoperated by EMG signals and arm motions.IEEE Trans-Robotic Automation 2003 Vol. 19. pp. 210-22.

3. [3] J. Zhao, et al. Levenbergmarquardt based neural network control for a five-fingered prosthetic hand. Proc. IEEE Int. Conf. Robotic Automation 2005. pp. 4482-87.

4. [4] S. Bitzer, et al. Learning EMG control of a robotic hand: Towards active prostheses. Proc. IEE Int. Conf. Robotic Automation 2006. pp. 2819-23.

5. [5] M. Zecca, et al. Control of multifunctional prosthetic hands by processing the electromyography signal. Crit. Rev. Biomed. Eng. 2002; Vol. 30. pp. 459-85.

6. [6] D. Nishikawa, et al. EMG prosthetic hand controller using real-time learning method. Proc. IEEE Int. Conf. Syst., Man, Cybern. 1999. pp. 153-158.

7. [7] S. Maier, et al. Surface EMG suffices to classify the motion of each finger independently. International Conference on Motion and Vibration Control 2008.

8. [8] K. Takahashi, et al. Remarks on hands-free manipulation using biopotential signals. ISIC/IEEE Int. Conf. Syst., Man Cybern 2007. pp. 2965-70

9. [9] Ambily Francis, et al. Multi-tasking EMG controlled robotic arm. IJARCCE 2017; Vol.6.

10. [10] A. V. Hill. The heat of shortening and the dynamic constant so muscle. Proc. Royal Society London B, Biol. 1938; Vol. 126. pp 136-195.

11. [11] Rutvij B. Mavani, et al. Design and working of myoelectric prosthetic arm. IJEDR 2014; Vol.2.

12. [12] V. Sumathi, et al. Acrylic prosthetic limb using EMG signal. International Journal of Engineering Inventions 2016: Vol.5. pp. $35-44$. Controlled Powered Exoskeleton Arm - Parameters Optimization.Proc.

13. [13] Ehab M. Faidallah, et al. Control and modelling a robotic arm via EMG and flex signals. International workshop on Research and Education in Mechatronics 2014: Vol.2. pp. 144-54.

14. [14] Anjali Raghavan, et al. EMG analysis and control of artificial arm. IJCI 2016; Vol.5.

15. [15] Mohan C, et al. DC motor control using EMG signal for prosthesis. IJECT 2011: Vol.2; Issue 2.

\section{AUTHORS PROFILE}

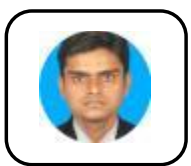

Dr.G.Vishnuvarthanan, born in 1986, has research stints in the avenues of medical image processing and artificial intelligence. $\mathrm{He}$ was awarded $\mathrm{PhD}$ in the year 2015 and bachelor's degree in Instrumentation and Control Engineering by 2007, and Master's Degree in VLSI by 2009. He has more than ten years of teaching and research experience and has his affiliation as Associate Professor with the Department of Biomedical Engineering of School of Bio and Chemical Sciences in the Kalasalingam Academy of Research and Education, Tamilnadu, India.

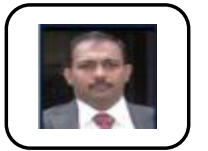

PallikondaRajasekaranMurugan, Born in Srivilliputhur, Virudhunagar District of Tamil Nadu in 1980, he had his schooling in the same town and graduated in Electronics and Instrumentation Engineering in 2001 from Shanmugha College of Engineering, Thanjavur and completed his M.Tech. degree in 2002 with second Rank in SASTRA University. He pursued his doctoral program in Anna University, Chennai. Starting as a Lecturer in 2003, he became Asst. Professor in 2008, Associate Professor in 2009 and Professor in 2012 in Kalasalingam Academy of Research and Education. He had a deep involvement in Bio-signal Processing research. His work on the Image Segmentation for identification of brain tumor and image reconstruction and compression using medical images for diagnosis. Over 150 B.Tech students, 75 M.Tech students, and 8 Doctorates stand testimony for his productivity in Image Processing, Wireless Sensor Networks, and Biomedical Instrumentation research. He has so far published more than 50 papers in national and international journals and conferences. $\mathrm{He}$ is a Fellow of Indian Society for Technical Education (ISTE), Institute of Electrical and Electronics Engineers (IEEE), Asia-Pacific Chemical, Biological \& Environmental Engineering Society (APCBEES), Institution of Engineers (India)(IE), International Association of Engineers (IAENG) and International Association of Computer Science and Information Technology (IACSIT).

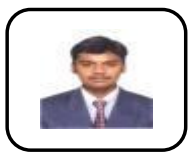

Sakthivel Sankaran received B-Eng. in Electronics and Communication Engineering, May 2013 and M. Tech in (Biomedical Engineering) at VIT University, Vellore, May 2015. Currently working as an Assistant Professor in Biomedical Engineering department at Kalasalingam Academy of Research and Education, Krishnankoil. His research interests are Biomechanics, Rehabilitation Engineering and Biomedical Instrumentation. He has so far published more than 09 papers in national and international journals and conferences. He is a Fellow of Indian Society for Technical Education (ISTE), Institute of Electrical and Electronics Engineers (IEEE), Biomedical Engineering Society of India (BMESI) (sakthivelsankaran92@gmail.com).

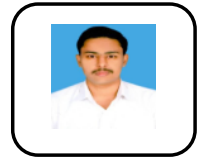

R. Madhan Balaji received B.E. in Biomedical Engineering, May 2015 and M.E. in Medical Electronics at Rajalakshmi Engineering College, Thandalam, Chennai, August 2017. Currently working as an Assistant Professor at Agni College of Technology, Chennai. His Research areas are Biomedical Instrumentation, Biomedical Nanotechnology and Biomedical Imaging.

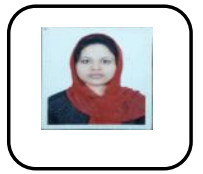

M. Sofia Fazilapursuing herB. Tech Biomedica Engineering at Kalasalingam Academy of Research and Education, Krishnankoil

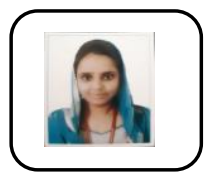

V. Sheik Hussain Beevipursuing herB. Tech Biomedical Engineering at Kalasalingam Academy of Research and Education, Krishnankoil

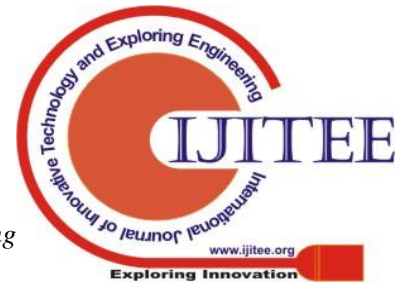


M. Marikanipursuing his B. Tech Biomedical Engineering at Kalasalingam Academy of Research and Education, Krishnankoil.

K. Ananda Lakshmipursuing her B. Tech Biomedical Engineering at Kalasalingam Academy of Research and Education, Krishnankoil. 